\title{
EXPLORATION OF THE EFFECT OF WORKERS' INFLUENCE NETWORK ON THEIR ABSENCE BEHAVIOR USING AGENT-BASED MODELING AND SIMULATION
}

\author{
Seungjun Ahn \\ Kyle Anderson \\ SangHyun Lee \\ Civil and Environmental Engineering \\ University of Michigan \\ Ann Arbor, MI 48109 USA
}

\begin{abstract}
Workers' absence behavior is not only determined by individuals' personal characteristics or situations, but also strongly affected by workgroup-level properties, such as social norms. This is because workers gather the how-to-behave information not only from formal rules, but also by interacting with their peers to obtain group and organizational approval. Despite the increasing attention being paid to the social control of workers' absence behavior in organizations, to date relatively little work has been done studying the impact of workers' influence networks, in which the social control takes place, on absence behavior. In this paper, we apply agent-based modeling and simulation (ABMS) to study the impact of workers' influence networks on absence behavior. Our simulation results suggest that small social networks of workers could be more effective than big networks in reducing absenteeism when the social control is active.
\end{abstract}

\section{INTRODUCTION}

Manpower is one of the most important resources in construction projects, making construction workers' timely attendance at project site is an indispensible condition for successful projects. Since construction workers usually work in teams (i.e., crews) when a construction worker is late or absent, other workers in the team have to compensate for the missing worker. The missing manpower, if not successfully compensated for by other team members, can damage project performance by interrupting workflow and impeding productivity, and in turn, can cause revenue loss (Hinze et al. 1985). Further, when the absence level in a workgroup is excessive, managers consider inputting substitutes to cover the missing roles, which can cause more indirect problems. For example, the substitutes of absentees may be inexperienced, less skilled or less familiar with the work environment, and therefore, workers are exposed to increased likelihoods of accidents, not to mention decreased productivity.

Recently an $8.5 \%$ absence rate was reported in large-scale industrial construction projects in Canada (Sichani et al 2011), and a 9.13\% average loss of productivity was observed in electrical construction projects in the US (Hanna et al. 2005). When construction managers deal with such high absence rates, they usually use individual-focused formal controls (e.g., penalties or incentives), but in many cases formal controls have been found ineffective (Sichani et al. 2011). It could be inferred from this that the formal controls did not sufficiently encourage workers' attendance motivations.

One factor in construction workers' absence decisions that has received relatively little attention is the effect of social control of absence behavior in workgroups. The absenteeism literature provides abundant evidence that shows that workers observe others' behavior and learn how-to-behave from their peers (Johns 2008). Social control of worker's behavior is exerted through the operation of social networks (Friedkin 2001). However, we do not have a clear understanding of the effect of construction workers' social influence networks on their absence behavior, which could provide insight into how to effectively and efficiently manage workers' absence behavior by using social controls in favor of management. 
Ahn, Anderson, and Lee

Therefore, we need to better understand how construction workers' absence behavior is affected by social influences via social networks

\section{BACKGROUND}

\subsection{Absence Culture and Social Control of Absence Behavior}

Gellatly and Luchak argue that absence behavior is partially controlled by absence-related belief (i.e., absence culture) at the group level (Gellatly and Luchak 1998). This shared absence-related belief is often called absence culture, which is defined as "the set of shared understandings about absence legitimacy in a given organization and the established 'custom and practice' of employee absence behavior and its control..." (p.136) (Johns and Nicholson 1982). Workers in organizations create absence culture on their own by observing their peers' absence behavior and internalizing the observed behavior (Bamberger et al. 2007; Iverson et al. 2003). Since the interaction with peers (e.g., observation) is the mechanism of the emergence and exertion of social absence norms, the shape of workers' influence networks in organizations can significantly affect workers' absence behavior. However, despite the increasing attention to the social control of workers' absence behavior in organizations, to date relatively little work has been done studying the impact of workers' influence networks on absence behavior.

The social influence theory attempts to explains how workers' influence networks can operate to modify attitudes and how those attitudes can converge by a sequence of interpersonal influences (Friedkin and Johnsen 2003). This theory can be applied to help explain workers' absence behavior. Individuals' attitudes toward absence in organization can be modified by other members' interpersonal influences, such as observing a peer's high absence rate. The interpersonal influences taking place via workers' influence networks can modify workers' absence-related belief and make their absence behavior in the organization assimilated over time. This is how absence cultures emerge from the influence networks.

\subsection{Previous Work}

Based on the social influence theory (Friedkin 2001) and the social cognitive theory of self-regulation (Bandura 1991), we have previously developed an agent-based model of construction workers' absence behavior (Ahn et al. 2013). This model represents an internal process that would produces one's absence behavior at each day. Figure 1 shows the concept of the agent behavior rule. Ahn et al. (2013) explains theoretical backgrounds for this model.

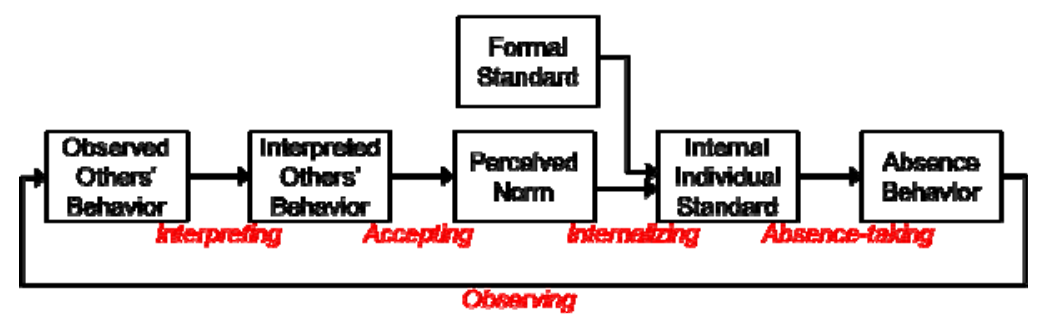

Figure 1. Absence behavior rule (adapted from Ahn et al. 2013)

Each worker in this model observes others' absence via social influence networks. The information of others' absence behavior is interpreted by the observing worker, and then the aggregated absence behavior of the worker's peers (who is connected by social influence networks) is perceived as a social norm. This step (termed as "accepting") is influenced by the worker's characteristic, social adaptation, which refers to "the degree of the worker's tendency to learn others' absence behaviors and follow them (Ahn et al. 2013)." The step is also influenced by salience, which refers to "the degree of the distinctiveness of an absence norm in the group (i.e., the degree of uniformity of the behavior of observed workers) (Ahn et al. 2013)." Therefore, the greater the social adaptation or salience, the worker tends to more quickly adapt his/her perceived norm to the observed others' behavior. Then, formal absence standards and the per- 
ceived norms are the sources of one's internal absence standard. Based on one's inclination to either of the standards (termed as formal rule awareness), one's internal absence standard is determined (termed as "internalizing"). Once an internal standard is set, absence behavior is produced to reduce the discrepancy between the internal absence standard and the current state (i.e., one's current absence rate). Ahn et al. (2013) also provides the computational rules for each step in this behavior model which can be used for computer simulations.

In this work, we conducted an experimental analysis by running simulations with the agent-based model to understand the impact of workers' characteristics that would affect the social learning on their absence behavior. More specifically, we explored the impact of the principal parameters in the modelsocial adaptation(i.e., how quickly workers adapt their perceived absence norm according to an observed others' behavior), formal rule awareness(i.e., how much more workers consider formal standards over perceived absence norms), and strictness of self-regulation (i.e., how strictly workers regulate their absence behavior ) - on the dynamics of workers' absence behavior. To fulfill this objective, we applied different values for these parameters and comparing the results. From this experimentation, we found that high social adaptation among workers can lead to the development of positive absence-related social norms, which can reduce the need for additional formal controls to manage workers' absence behavior. Particularly, the emergence of social norms in favor of management was observed when workers have strict self-regulation (i.e., the degree to which workers regulate their absence behavior based on the individual standards) in the simulation.

The present paper is also in this vein of performing experiments with the model to extend our knowledge of workers' absence behavior under influences of social norms. The objective of this paper is to explore the effect that network degree, number of social connections between workers, has on absence behavior. More specifically, we aim to test the impact of different levels of social connections across crews (i.e., average number of connections across crews) - which can be changed by interventions taken by management - on the reduction of the absence rate. This paper will proceed to describe the simulation model, the experimental design and simulation results. Finally, this paper concludes with a discussion on the results.

\section{METHOD}

In this paper, we use the same agent behavior rules that we presented in the previous paper. In the agentbased model, workers can observe their peers' absence when it occurs. The peers are the ones who are connected with the workers by an influence network in the organization. The observed behavior is remembered and interpreted in each worker's mind, and shapes each individuals' perception of social absence norms. The perceived absence norm and the formal absence standard are the sources of individual workers' internal standard of absence behavior. Based on the internal standard, workers regulate their own absence behavior.

The main independent variable in the experiment presented in the current study is the number of connections across crews. Since construction workers usually work in teams, it is reasonably assumed that workers in their own crew all know each other and can observe every team member's behavior, so each is connected with everyone in their crew. In the network theory terminology, this type of network is called a clique (Newman 2010). Outside of their clique, construction workers should have relatively fewer connections across crews, since members of different crews have fewer opportunities to interact. However, if managers emphasize team processes at the entire project level and promote cognitive attributes such as a team mental model and team situation awareness across crews (Mitropoluos and Mermarian 2012), workers' influence networks across crews can significantly increase in number. Therefore, we assumed that various degrees of influence networks across crews are possible in construction project organizations.

To explore the impact of the degree of workers' influence networks, we simulated a 100 -worker project organization consisting of 10 crews, each of which has 10 workers. In the simulation, the formal absence standard is 1 day per month, but workers are initialized with a high absence standard ranging from 1 to 5 days per month, which simulates a problematic scenario. Then, we applied different degrees of in- 
fluence network across crews, such as $0 \%, 10 \%, 20 \%, 30 \%, 40 \%, 50 \%$ and $60 \%$. These inputs determine the probability of a connection between workers from different crews. Therefore, " $60 \%$ " means a worker would approximately know $60 \%$ of all the workers in the organization beyond their own crew members. Two different settings of the model parameter that represents the strictness of self-regulation were used in this study because in our previous experiments this parameter had a critical impact on workers' absence behavior. Also, workers' formal rule awareness has been set as low and social rule awareness as high in order to highlight the impact of social control on absence behavior. In each simulation run we collect absence rates at the 100th day and the 200th day in the simulation to see the change in the absence rate, and we replicate the simulation with each setting 100 times to statistically analyze simulation results

\section{SIMULATION RESULTS}

The figures below show the simulation results. Each box plot in the figures shows the distribution of data that is produced from simulations with the same setting. As shown in the Figure 1-(a) and (b), on average the absence rate decreases the most when the across-crews network degree is $0 \%$ (i.e., workers are influenced only by members of their own crew) in the simulation. With this setting, the mean absence rate decreases from 2.5 day/month (initially) to 1.0 day/month (at the 100th day) and to 0.85 day/month (at the 200th day). As the across-crews network degree increases (i.e., workers have connections with the workers of other crews too, so workers in general observe a larger sample of others' behavior), the reduction of absence rate decreases.

The comparison between Figure 2-(a) and (b) indicates that the reduction of absence rate led by social control is quick when the network degree is rather low. In other words, the simulation results show that small, segmented networks may be more effective for reducing absence rate by social control because the reduction of absence rate can occur more quickly.

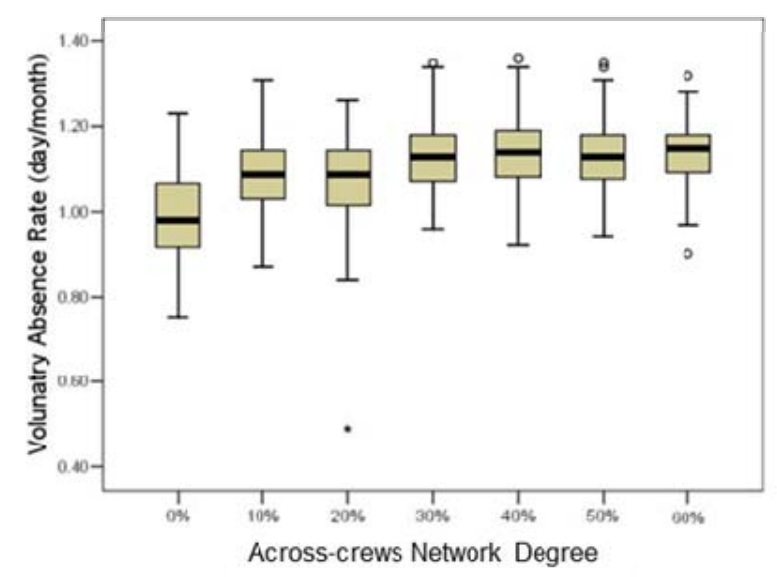

(a) at $100^{\text {th }}$ day

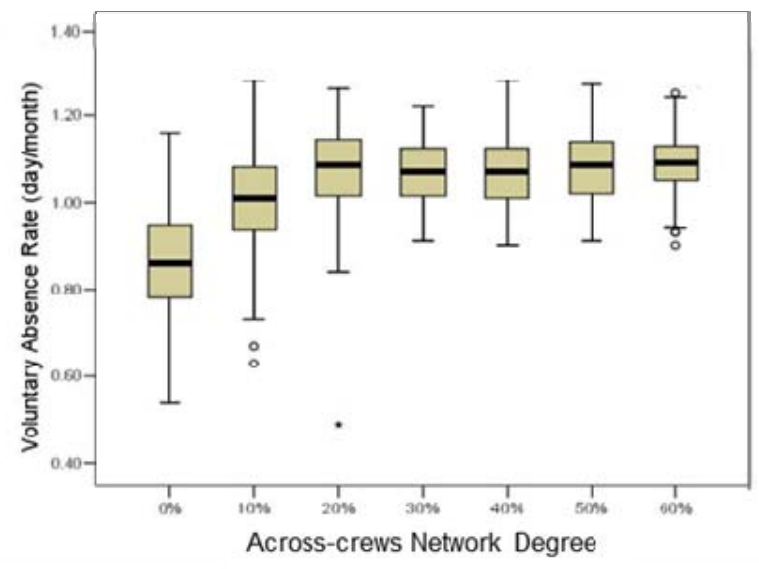

(b) at $200^{\text {th }}$ day

Figure 2. Absence rate change (when workers have strict self-regulation)

As shown in Figure 3-(a) and (b), in the case of less strict self-regulation, altering the network degree has little effect on the absence rate both at the 100th and the 200th day in the simulation.

\section{DISCUSSION}

The simulation results might seem counterintuitive, because it tells us that when workers have connections with more workers, the reduction of workers' absenteeism led by the emergence of positive social control is less. In other words, small, segmented social influence networks were more effective in reducing absence rate over time by social control in our simulations. This is believed to be because small net- 
works are more dynamic. The dynamic relationship between workers' behavior and perception is the mechanism of reducing absence rates by social control.

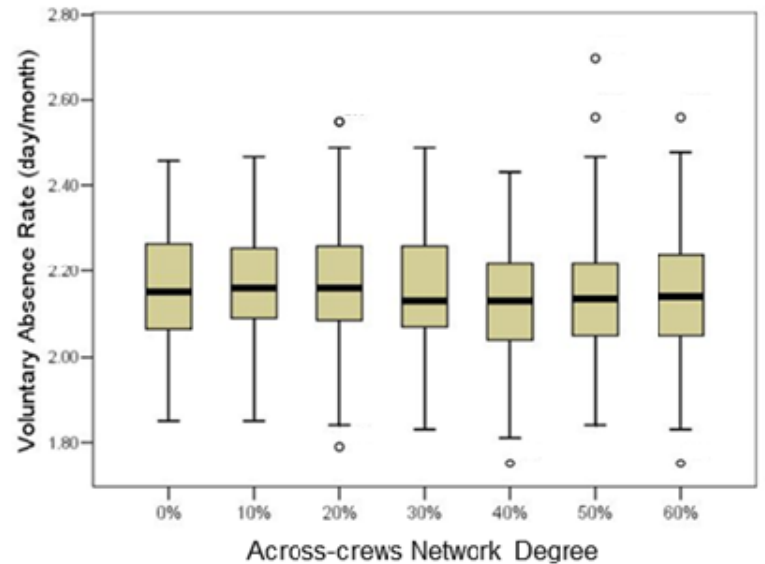

(a) at $100^{\text {th }}$ day

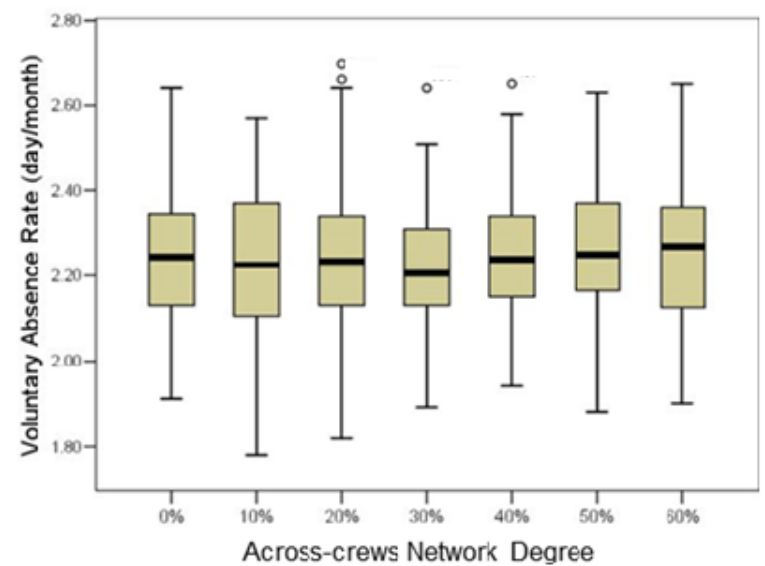

(b) at $200^{\text {th }}$ day

Figure 3. Absence rate change (when workers have less strict self-regulation)

In other words, workers observe a low absence rate of their peers and they also present a low absence rate, and so on. This mechanism causes the reduction of absence rates to occur rather quickly in small networks. Thus, small networks could be more effective in reducing absence behavior with positive social norms. When the network is big, the overall absence behavior changes slowly, so may not be effective in reducing absence behavior considering the duration of construction projects.

When workers have less strict self-regulation, the social control does not positively contribute to the reduction of absence rate. We could observe that the absence rate even increased slightly between the 100 th day and the 200th day in the simulation (i.e., prevalence of bad social norms). When workers have less strict self-regulation, the difference in the network degree did not make much difference in absence rate change in our observation of the simulation results. This may be because the increase in absence rate caused by the low self-regulation (i.e., workers do not put much efforts to control their behavior whatever the internal standard is) occurs very quickly, at the 100th day in the simulation the absence rate could already have reached a "near-peak".

\section{CONCLUSIONS}

We applied ABMS to study the impact of workers' influence networks on absence behavior. From our simulation results, we found that if social control works in favor of management and so reduces absence rate in the organization over time, small networks can be more effective than large, entire organizationwide network at reducing absenteeism. Therefore, if a project is currently suffering from absenteeism and wants to reduce absence rates at least partially by social control, project managers should consider two things: how to improve the strictness of self-regulation among workers (see Ahn et al. (2013) for more discussion on methods) and how to stimulate the mechanism of social control on absence behavior by shaping workers' influence networks. Our recommendation is to avoid promoting excessively large group networks of workers. This is because the positive, dynamic social control can more easily emerge in cohesive small groups (e.g., crew). Therefore, project managers may want to promote social gatherings at the crew level by providing more chances to interact within their own workgroups. An example of this approach is using team feedback of crew members' job performance.

We expect that the result of our research presented in this paper will help construction organizations better understand the impact of workers' influence networks on reducing absenteeism in projects. However, since the simulation model was created with many assumptions, this research warrants future efforts to 
Ahn, Anderson, and Lee

validate the modeling assumptions and findings with empirical observations. Lastly, more extensive sensitivity analyses are needed to create more comprehensive knowledge of the operation of workers' influence network and its impact on workers' absence behavior.

\section{ACKNOWLEDGMENTS}

The work presented in this paper has been supported by the National Science Foundation Award (SES 1127570).

\section{REFERENCES}

Ahn, S., S. Lee and R. P. Steel. 2013. "Effects of Workers' Social Learning: Focusing on Absence Behavior." Journal of Construction Engineering and Management, ASCE, in press.

Bamberger, P., and M. Biron. 2007. "Group norms and excessive absenteeism: The role of peer referent others." Organizational Behavior and Human Decision Processes, 103(2):179-196.

Bandura, A. 1991. "Social cognitive theory of self-regulation." Organizational Behavior and Human Decision Processes, 50:248-287.

Friedkin, N. E., and E. C. Johnsen. 2003. "Attitude change, affect control, and expectation states in the formation of influence networks." Advances in Group Processes, 20(3):1-29.

Friedkin, N. 2001. "Norm formation in social influence networks." Social Networks, 23(3):167-189.

Gellatly, I. R., and A. A. Luchak. 1998. "Personal and Organizational Determinants of Perceived Absence Norms." Human Relations, 51(8):1085-1102.

Hanna, A. S., C. L. Menches, K. T. Sullivan, and J. R. Sargent. 2005. "Factors Affecting Absenteeism in Electrical Construction." Journal of Construction Engineering and Management, 131(11):1212-1218.

Hinze, J., M. Ugwu, and L. Hubbard. 1985. "Absenteeism in Construction Industry." Journal of Management in Engineering, 1(4):188 - 200.

Iverson, R. D., D. M. Buttigieg, and C. Maguire. 2003. "Absence culture: the effects of union membership status and union-management climate." Relations Industrielles/Industrial Relations, 58(3):483515.

Johns, G. 2008. "Absenteeism and presenteeism: Not at work or not working well." The Sage handbook of organizational behavior, 1:160-177.

Johns, G. and N. Nicholson. 1982. "The meaning of absence: new strategies for theory and research." In B. Staw, \& L. L. Cummings (Eds.), Research in organizational behavior, 4:127-172. Greenwich, CT: JAI Press.

Mitropoulos, P. and B. Memarian. 2012. "Team Processes and Safety of Workers: Cognitive, Affective, and Behavioral Processes of Construction Crews." Journal of Construction Engineering and Management, 138(10):1181-1191.

Newman, M. 2010. Networks: an introduction. Oxford University Press, Inc..

Sichani, M. S., S. Lee, and A. R. Fayek. 2011. "Understanding construction workforce absenteeism in industrial construction." Canadian Journal of Civil Engineering, 38(8):849-858.

\section{AUTHOR BIOGRAPHIES}

SEUNGJUN AHN is a Ph.D candidate in the Civil and Environmental Engineering Department of the University of Michigan. His research focuses on modeling organizational behavior of construction workers and impact of norms on the workers' behavior and predicting the system-level effectiveness of human resource management in construction projects. Along with pursuing his $\mathrm{PhD}$, he is working toward a Certificate of Graduate Studies in Complex Systems offered by the Center for the Study of Complex Systems at the University of Michigan. His email address is esjayahn@umich.edu. 
KYLE ANDERSON is a Ph.D student in the Civil and Environmental Engineering Department of the University of Michigan. He is interested in behavioral approaches to reduce energy consumption in the built environment. In particular, he investigates how we can promote generalizable and durable changes in occupant behavior. His email address is kyleand@umich.edu.

SANGHYUN LEE is an Assistant Professor at the University of Michigan. Before joining the faculty there, Dr. Lee worked as an Assistant Professor at the University of Alberta, Canada, from 2007 to 2010. He earned a Ph.D. in Construction Management and Information Technology from MIT, and worked at CRA International as a consultant and participated in numerous international construction projects while teaching at MIT (2006-2007). Dr. Lee's main research interest is the understanding and management of construction dynamics through the design and development of mechanisms, models, and systems that enhance proactive management of mega construction projects. His e-mail is shdpm@umich.edu. 